\title{
Article \\ Effects of Nitrogen plus Sulfur Fertilization and Seeding Density on Yield, Rheological Parameters, and Asparagine Content in Old Varieties of Common Wheat (Triticum aestivum L.)
}

\author{
Quadratullah Soofizada ${ }^{1}$, Antonio Pescatore ${ }^{1}$ (D), Lorenzo Guerrini ${ }^{2}{ }^{(D)}$, Carolina Fabbri $^{1}$, Marco Mancini ${ }^{1}$, \\ Simone Orlandini ${ }^{1}$ and Marco Napoli ${ }^{1, *(D)}$
}

check for

updates

Citation: Soofizada, Q.; Pescatore, A.; Guerrini, L.; Fabbri, C.; Mancini, M.; Orlandini, S.; Napoli, M. Effects of Nitrogen plus Sulfur Fertilization and Seeding Density on Yield, Rheological Parameters, and Asparagine Content in Old Varieties of Common Wheat (Triticum aestivum L.). Agronomy 2022, 12, 351. https:// doi.org/10.3390/agronomy12020351

Received: 17 December 2021

Accepted: 28 January 2022

Published: 30 January 2022

Publisher's Note: MDPI stays neutral with regard to jurisdictional claims in published maps and institutional affiliations.

Copyright: (C) 2022 by the authors. Licensee MDPI, Basel, Switzerland. This article is an open access article distributed under the terms and conditions of the Creative Commons Attribution (CC BY) license (https:// creativecommons.org/licenses/by/ $4.0 /$ )
1 Department of Agriculture, Food, Environment and Forestry (DAGRI), University of Florence, Piazzale delle Cascine 18, 50144 Firenze, Italy; qudratullah.soofizada@unifi.it (Q.S.); antonio.pescatore@unifi.it (A.P.); carolina.fabbri@unifi.it (C.F.); marco.mancini@unifi.it (M.M.); simone.orlandini@unifi.it (S.O.)

2 Department of Land, Environment, Agriculture and Forestry (TESAF), University of Padua, Viale dell’Università, 16, 35020 Legnaro, Italy; lorenzo.guerrini@unipd.it

* Correspondence: marco.napoli@unifi.it

\begin{abstract}
Numerous epidemiological studies have highlighted the positive effects on health of wholegrain bakery products made from 'old' common wheat (Triticum aestivum L.) varieties. However, 'old' common wheat varieties display poor rheological properties, and there is limited information on its free asparagine (ASN) content, the main precursor to acrylamide during the baking process. This paper evaluates the effects of two seeding density levels (SD: 200 and 350 seed $\mathrm{m}^{-2}$ ), three nitrogen levels (NL: 35, 80 and $135 \mathrm{~kg} \mathrm{~N} \mathrm{ha}^{-1}$ ), and two sulfur levels (SL: 0 and $6.4 \mathrm{~kg} \mathrm{~S} \mathrm{ha}^{-1}$ ) towards improving the grain yield (GY), rheological characteristics, and ASN content of 14 'old' common wheat varieties. SL and SD treatments significantly increased GY without decreasing the protein content (PC), while NL significantly increased the PC without affecting GY. The dough strength $(\mathrm{W})$ increased significantly with increasing SL and NL but was significantly reduced with increasing SD. ASN significantly increased by $111 \%$ as NL increased from 35 to $135 \mathrm{~kg}^{-1}$, while ASN significantly decreased by $85.1 \%$ with the SL treatment. The findings show that $135 \mathrm{~kg} \mathrm{~N} \mathrm{ha}^{-1}$ combined with $6.4 \mathrm{~kg} \mathrm{~S} \mathrm{ha}^{-1}$ can improve the technical performance of 'old' wheat wholegrain flours while maintaining the ASN as low as possible.
\end{abstract}

Keywords: old common wheat varieties; agronomic treatments; sulfur fertilization; free asparagine; rheological properties

\section{Introduction}

Common wheat (Triticum aestivum L.) is one of the most important cereals worldwide for both human and livestock consumption, contributing towards enhancing the global economy [1,2]. Common wheat production amounted to $761 \mathrm{Mt}$ in 2020 [3] and provides protein for the nutrition of both humans and livestock, estimated at around $60 \mathrm{Mt} \mathrm{y}^{-1}$, as reported in Shewry (2009) [4]. After the Green Revolution, common wheat production increased, attributable to intensive fertilizer use and the breeding of cultivars, respectively, characterized by increased tolerance to diseases and pests, higher nutrient use efficiency, as well as a higher protein production per hectare, with a gluten composition suitable for industrial processing [5-9]. Conventionally, common wheat cultivars registered before the late 1960s are referred to as 'old', while those registered coinciding with the period of the Green Revolution are referred to as 'modern' [7].

In the past decades, 'old' common wheats varieties have been reintroduced, and many local micro-economies have been developed around 'old' cultivars [7,10]. In fact, the increase in pollution and food security problems has led us to reconsider common 
wheat production in terms of not only productivity but also of environmental and human health impacts [11]. Interest in low impact and sustainable agricultural practices, combined with functional (health-promoting) products, has permitted the rediscovery of 'old' common wheat varieties, considered to be more suited to unfavorable environmental factors and with improved functional value in comparison to the 'modern' varieties [12]. Numerous epidemiological studies have highlighted the positive effects on health and disease prevention of bread and other bakery products made from 'old' varieties [13,14]. In particular, the production of wholegrain bakery products is recommended as most bioactive compounds, associated with health benefits, are concentrated in the bran and aleurone layers, respectively $[15,16]$. However, although the aleurone layer also contains good quality free amino acids and proteins, it also stores free ASN, which is the predominant precursor of acrylamide formation in wholegrain bakery products [16,17]. As acrylamide is classified as a neurotoxin and "probably carcinogenic to humans" by the International Agency for Research on Cancer [18], free ASN concentration in grain should be monitored and maintained as low as possible. Corol et al. (2016) [19] found the free ASN contents in 150 genotypes of common wheat, ranging from 0.32 to $1.56 \mathrm{mg} \mathrm{g}^{-1}$ of dry matter (corresponding to $2.4-11.8$ micromoles $\mathrm{g}^{-1}$ of dry matter) in wholemeal wheat flours. The 'old' cultivars are characterized by poor efficiency in converting assimilated nitrogen $(\mathrm{N})$ to grain protein; this may contribute to an increased accumulation of ASN [20]. Furthermore, the grain ASN content may increase in relation to stress conditions such as waterlogging, drought, and plant diseases, as well as either nutrient excesses or deficiencies [21]. Of all the essential nutrients applied in the field, $\mathrm{N}$ is the most important for vegetative crop growth, productivity, and grain quality, thereby affecting plant development [22]. Sulfur (S) is an essential element for wheat nutrition, and S deficiency significantly affects the production and quality of wheat [23]. Interestingly, it was observed that ASN formation was correlated positively with $\mathrm{N}$ availability [24] but was increased in the presence of $\mathrm{S}$ deficiencies [20]. In this context, Wilson et al. (2020) detected free ASN concentrations ranging from 21.0 to 41.4 micromoles $\mathrm{g}^{-1}$ in S-deficient conditions. Aside from the effects on ASN, S affects not only $\mathrm{N}$ utilization and grain quality [25] but also plays an important role in baking quality [7]. Thus, optimized $\mathrm{S}$ and $\mathrm{N}$ fertilization practices can be implemented to reduce the ASN concentration in wholegrain common wheat and, consequently, act towards reducing the health concern of acrylamide in the baked products [26].

Despite the increased interest in old varieties for functional benefits and low input agricultural practices, these varieties are also usually characterized by a low dough strength (W) and an unbalanced ratio between dough tenacity and dough extensibility (P/L) compared to modern varieties. These rheological parameters render old varieties more difficult to bake [7]. In order to improve the rheological properties of both old common and durum varieties, research on fertilizer supplements is currently being investigated $[7,27]$.

While ASN content in common wheat grain has been studied extensively on a global scale $[20,21,28,29]$, only limited information on ASN concentrations in 'old' cultivars is available [30]. Given the increasing importance of 'old' cultivars and the success of crop management strategies in reducing ASN content in 'modern' cultivars, to the best of our knowledge, there is no work specifically focused on reducing the ASN concentration in the grain of 'old' cultivars. To address this aspect, the present study is aimed at investigating grain yield, dough rheology, and ASN concentration of 14 "old" Italian Triticum aestivum L. varieties in response to varying seeding density (SD) as well as $\mathrm{N}$ and $\mathrm{S}$ fertilization rates. The objective is to simultaneously evaluate the capacity of these agronomical practices in improving the technical performance of the dough whilst maintaining the lowest levels of ASN. 


\section{Materials and Methods}

\subsection{Field Experiment}

The experimental field trials were conducted at the demo-farm "Tenuta di Cesa" in Marciano della Chiana, Tuscany (Lat. 43.3095; Lon. 11.8264; $246 \mathrm{~m}$ asl) from September 2017 to July 2019 under rainfed conditions on an alkaline clay-loam soil (Table 1).

Table 1. Soil properties.

\begin{tabular}{cc}
\hline Soil Parameters & Value \\
\hline Sand (\%) & 37 \\
Clay (\%) & 34 \\
Silt (\%) & 27 \\
pH & 8.13 \\
Organic matter (\%) & 0.88 \\
Total N (\%) & 0.03 \\
Olsen available P (mg kg $\left.{ }^{-1}\right)$ & 0.42 \\
Available S (mg kg $\left.{ }^{-1}\right)$ & 3.3 \\
\hline
\end{tabular}

The soil was characterized by a low organic matter content and low nutrient availability. In particular, the soil was both phosphorous- and sulfur-deficient, with less than $10 \mathrm{mg} \mathrm{kg}^{-1}$ available P [31] and S [32], respectively. Fourteen old Italian varieties of common wheat (Triticum aestivum L.) were investigated. The varieties were: Acciaio (AC), Andriolo (AN), Autonomia A (AU_A), Autonomia B (AU_B), Bianco Nostrale (BI), Frassineto 405 (FR), Gentil Bianco (GB), Gentil Rosso (GR), Gentil Rosso Aristato (GR_A), Gentil Rosso Mutico (GR_M), Inallettabile (IN), Mentana (ME), Sieve (SI), and Verna (VE) (Table 2).

Table 2. Release year and origin for the wheat cultivars used in this study. Data were obtained from the website of the seed bank in the Tuscany Region [33].

\begin{tabular}{ccc}
\hline Variety & Year of Release & Origin \\
\hline AC & 1950 & Selection of "Mara", in turn, selection of "Frassineto 405" \\
AN & 1933 & Selection of the local landrace "Andriolo" \\
"Fus_A & 1938 & "Frassineto 405" $\times$ "Mentana" \\
AU_B & 1930 & Selection of the local landrace "Bianco Nostrale" \\
BI & 1927 & Pureline selection of "Gentil Rosso" \\
FR & 1932 & Local landrace dating back to the late 19th century \\
GB & 1900 & Local landrace dating back to the late 19th century \\
GR & 1900 & Selection of the local landrace “Gentil Rosso" \\
GR_A & 1900 & Selection of the local landrace "Gentil Rosso" \\
GR_M & 1900 & Selection of "Hatif Inversable" \\
IN & 1920 & ("Wilhelmina" $\times$ "Rieti 21") $\times$ "Akakomugi" \\
ME & 1913 & "Est Mottin 72" $\times$ "Bellevue II" \\
SI & 1960 & "Est Mottin 72" $\times$ "Mont Calme" \\
VE & 1953 &
\end{tabular}

Five of the old varieties in the trial were derived from the older varieties that were used as parental material. These included AU_A and AU_B, which were derived from crossing ME $\times$ FR, and FR, GR_A and GR_M, derived from the selection of the GR landrace. The genealogy and release dates of the varieties were obtained from the website of the seed bank in the Tuscany Region [33].

The 14 wheat genotypes (Gen) were evaluated during two growing seasons (Y) with 12 agronomic treatments comprising two seeding densities (SD) (200 and $350 \mathrm{~kg} \mathrm{seed} \mathrm{m}^{-2}$, namely, SD200 and SD350, respectively), three nitrogen fertilization rates (NL) (35, 80 and $135 \mathrm{~kg} \mathrm{~N} \mathrm{ha}^{-1}$, namely, NL35, NL80, NL135, respectively), and two sulfur fertilization rates (SL) (0 and $6.4 \mathrm{~kg} \mathrm{~S} \mathrm{ha}^{-1}$, namely, SL0 and SL6.4, respectively) (Figure 1). The experiment was established as a strip-plot design with three replicate blocks per year. Gen was arranged 
in vertical strips as the main plot, SD was assigned to the vertical sub-plots, SL was applied horizontally in sub-sub-plots, and, lastly, NL was assigned to horizontal sub-sub-subplots, respectively. Each sub-sub-subplot was $14.4 \mathrm{~m}^{2}$ (width of $1.44 \mathrm{~m}$ and length of $10 \mathrm{~m}$ ).

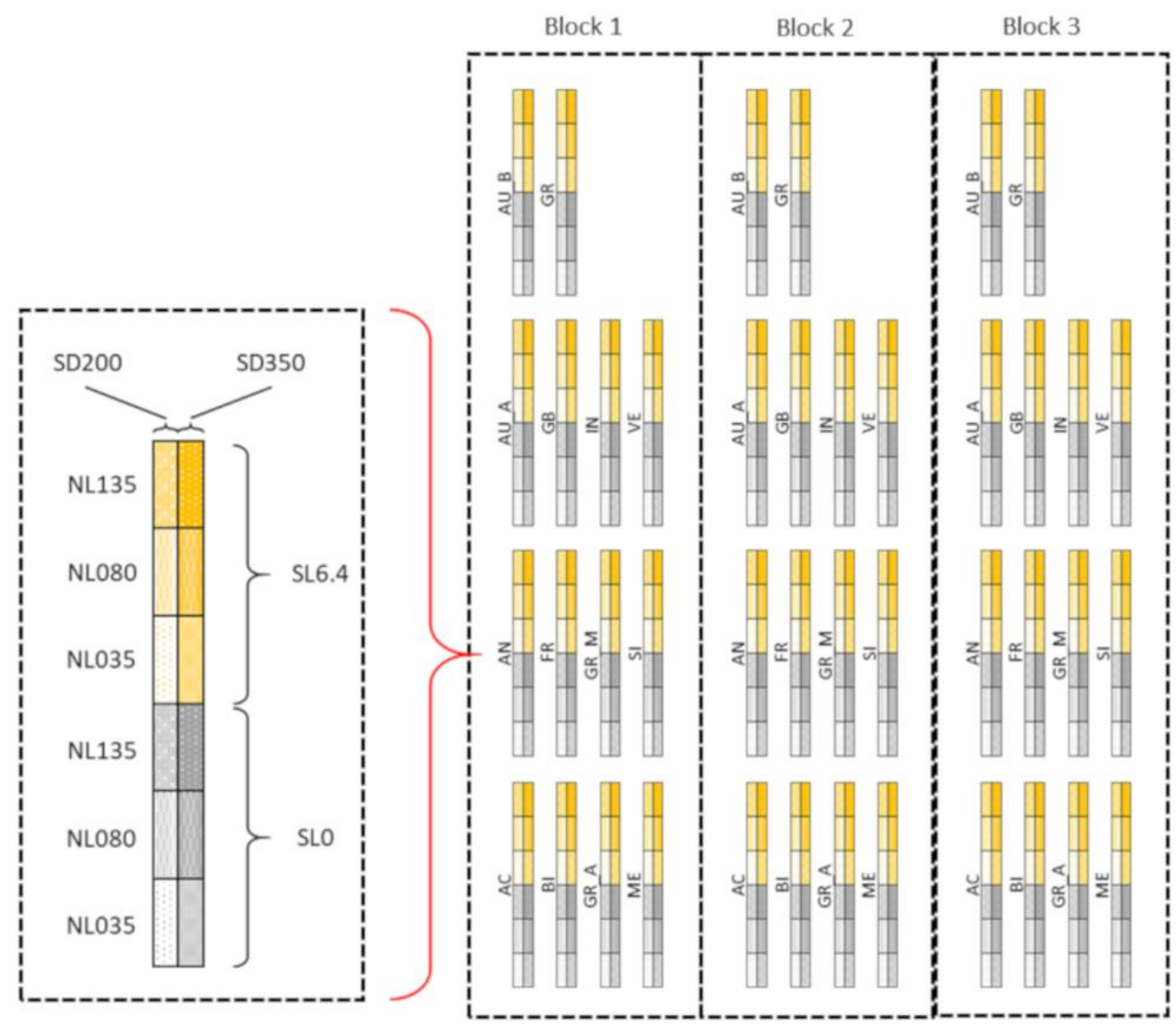

Figure 1. Experimental design and plot layout of trials (not in scale); On the left, the plot layout for each variety: SD200 and SD350 represent seeding density of 200 and $350 \mathrm{~kg}$ seed m${ }^{-2}$, respectively; NL35, NL80 and NL135 represent the three nitrogen fertilization rates of 35, 80 and $135 \mathrm{~kg} \mathrm{~N} \mathrm{ha}^{-1}$, respectively; SL0 and SL6.4 represent the two sulfur fertilization rates of 0 and $6.4 \mathrm{~kg} \mathrm{~S} \mathrm{ha}^{-1}$, respectively. On the right is the disposition of plots within the blocks for different varieties.

Soil tillage was carried out to a depth of $0.4 \mathrm{~m}$ with a moldboard plow in both September 2017 and 2018, followed by a tandem disk harrow (0.1 $\mathrm{m}$ depth) to break the clods. Before seeding, $174 \mathrm{~kg} \mathrm{ha}^{-1}$ of triple superphosphate $\left(\mathrm{P}_{2} \mathrm{O}_{5}: 46 \%\right)$ was broadcasted and immediately incorporated into the soil by means of a tandem disk harrow $(0.05 \mathrm{~m}$ depth). The seeding was performed on 20 November and 15 November in the first and second year, respectively. Nitrogen application was implemented over three distinct periods. Initially, 20\% nitrogen was broadcasted at seeding as ammonium nitrate (N: $26 \%$ ). Thereafter, $40 \%$ was spread at tillering as ammonium nitrate (N: $26 \%$ ), with a final $40 \%$ at the beginning of the stem elongation as urea (N: 46\%). As suggested in Guerrini et al. (2020), in S6.4, a total of $6.4 \mathrm{~kg} \mathrm{~S} \mathrm{ha}^{-1}$ was distributed at booting by spraying a solution containing $20 \mathrm{~g} \mathrm{~L}^{-1}$ of wettable sulfur powder $\left(80 \%\right.$ a.i.; Thiovit Jet $80 \mathrm{WG}^{\circledR}$, Syngenta, Basel, Switzerland). At tillering, a broadleaf herbicide treatment was performed by distributing Manta Gold (Syngenta, Basel, Switzerland) at a dose of $2.5 \mathrm{~L} \mathrm{ha}^{-1}\left(60 \mathrm{~g} \mathrm{~L}^{-1}\right.$ fluroxipir acid, 23.3 Clopyralid, and $266.7 \mathrm{~g} \mathrm{~L}^{-1} \mathrm{MCPA}$ acid). The monocot weeds were removed from each plot by performing manual weeding at tillering and at stem elongation. In both growing seasons, no crop damage by weeds, insects, or diseases was observed. Common wheat was harvested at commercial maturity (grain moisture $<13 \%$ ) on 12 July 2018 and 
5 July 2019. For each sub-sub-subplot, the grain biomass was calculated to determine the grain yield per hectare $\left(\mathrm{GY}, \mathrm{t} \mathrm{ha}^{-1}\right)$.

\subsection{Meteorological Conditions}

The climatic conditions were typically Mediterranean, with average daily temperatures around $13{ }^{\circ} \mathrm{C}$ and approximately $750 \mathrm{~mm}$ of rain per year, mostly concentrated in autumn and spring, as well as the dry summer period (Figure 2).

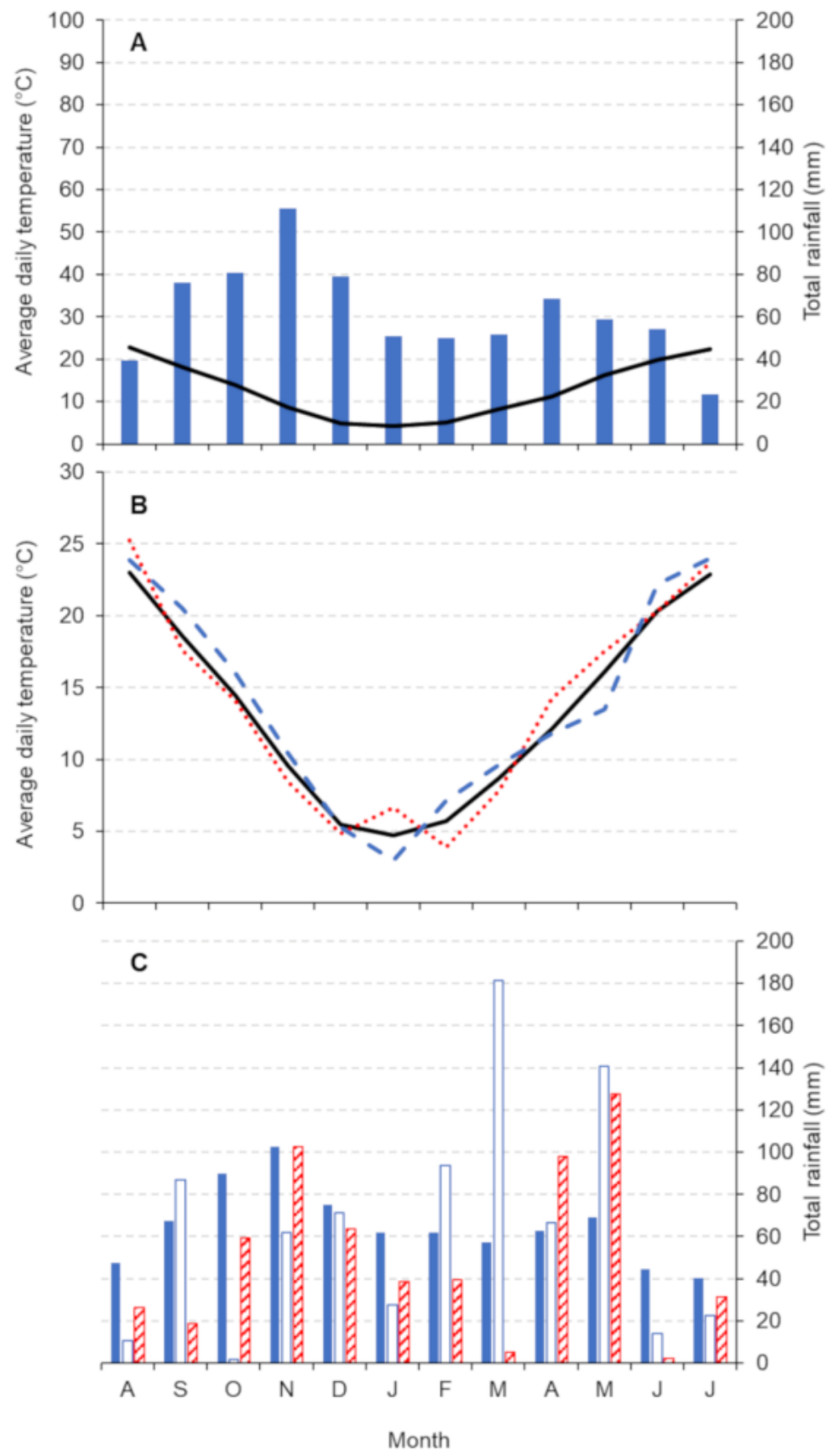

Figure 2. (A) Walter-Lieth climate diagram of the study site (data 2001-2020), with monthly daily average temperature $\left({ }^{\circ} \mathrm{C}\right.$, black continuous line) and monthly average rainfall amount ( $\mathrm{mm}$, histograms). (B) Comparison of the monthly daily average temperature ( $\mathrm{mm}$ ) measured during 2001-2020 (continuous line), 1st growing season (dashed line) and 2nd growing season (dotted line); (C) comparison of the monthly rainfall amount (mm) measured during 2001-2020 (color-filled histograms), 1st growing season (hollow histograms) and 2nd growing season (diagonal-filled histograms). 
The average temperature pattern during both growing seasons was consistent with the long-term temperature pattern. However, the average temperature values across the first and second growing seasons (13.7 and $13.9^{\circ} \mathrm{C}$, respectively) were higher than the long-term average $\left(13.0^{\circ} \mathrm{C}\right)$. In both years, rainfall distribution data fluctuated significantly with respect to the long-term rainfall pattern.

During the first growing season, excess rainfall was recorded from February to May, corresponding to the tillering to flowering phenological stage of common wheat. Then, a shortage of rainfall was experienced in June, the month coinciding with grain filling. The average temperature values at flowering and grain filling in the spring season of 2018 were slightly warmer than the long-term averages by about 1.2 and $0.5^{\circ} \mathrm{C}$, respectively.

During the second growing season, excess rainfall was recorded in April and May (from the booting to flowering phenological stage of common wheat), while a rainfall shortage was experienced in March, coinciding with stem elongation, as well as June. During the summer months of 2019, the daily average temperature at flowering was lower than the long-term average by $2.7^{\circ} \mathrm{C}$, while the average temperature at grain filling exceeded the long-term average by $2.4{ }^{\circ} \mathrm{C}$. Therefore, between May and June 2019, there was a temperature increase of $8.7^{\circ} \mathrm{C}$, which could have resulted in stress for the plants during both the initiation and grain-filling phases.

\subsection{Analysis of Kernels and Dough}

The 1000 kernel weight (TKW, g 1000-1 seeds) and hectoliter weight (HW, $\mathrm{kg} \mathrm{hL}^{-1}$ ) were determined according to ISO 7971-1 (2009) and ISO 520 (2010) [34,35]. For each treatment, wholemeal flour samples were obtained by milling kernel samples in a grinder with a $0.5 \mathrm{~mm}$ screen (Cytolec 1093 lab mill, FOSS Tecator, Hoganas, Sweden), as reported in Guerrini et al. (2020) [7] and Žilić et al. (2011) [36]. The wholemeal flour samples (5 mg) were analyzed with a CHNS analyzer (CHN-S Flash E1112, Thermo-Finnigan LLC, San Jose, CA, USA) to determine total nitrogen percentage and then converted to total protein percentage (PC, \%) by multiplying by 5.7, according to ICC Standard 167 (2000) [37]. The protein yield per hectare (PY, $\mathrm{kg} \mathrm{ha}^{-1}$ ) was calculated as the product of GY by PC. The ASN concentration in wholegrain flour (ASN, micromoles $\mathrm{g}^{-1}$ ) was determined using an enzymatic method (K-ASNAM L-Asparagine/L-Glutamine/Ammonia kit; Megazyme, IL, USA) followed by spectrophotometric quantification (340 nm) using a Lambda 20 spectrophotometer (PerkinElmer Waltham, MA, USA), as reported by Lecart et al. (2018) [38].

Dough rheology was performed according to ISO 27971 (2015) [39]. Briefly, wholegrain flour (250 g) was mixed in the Chopin alveograph chamber with a $\mathrm{NaCl}$ solution $(2.5 \%$ $w / w)$ for $8 \mathrm{~min}$ without adding yeast. The resulting dough was extruded and allowed to rest for $20 \mathrm{~min}$ before performing the alveograph parameters: the ratio between dough tenacity and dough extensibility $(\mathrm{P} / \mathrm{L})$ and the dough strength $\left(\mathrm{W} ; 10^{-4} \mathrm{~J}\right)$. TKW, HW, and PC were determined for each sub-sub-subplot, while ASN, W and P/L were determined for each treatment on a bulk from the three replicates.

\subsection{Statistical Analysis}

Data were analyzed using a mixed model analysis of variance. Both years' trial data were analyzed together. Data analysis was carried out in R studio (software version 1.1.456). A 4-way ANOVA was applied to determine the main effect of the four agronomical factors with their interactions. Significance was determined as: ${ }^{*}=0.05,{ }^{* *}=0.01$, $* * *=0.001$, n.s. $=$ not significant. Differences between averages were compared for significance by means of the Tukey honest significant difference (Tukey HSD) test $(p<0.05)$.

\section{Results}

\subsection{Agronomic Traits and Kernel Analyses}

The Y was the dominant factor for GY, followed by SL, Gen and SD, while the NL did not significantly affect GY (Table 3). 
Additionally, GY was significantly affected by the interaction $\mathrm{Y} \times \mathrm{SD}$, whilst no interactions between $\mathrm{Y} \times \mathrm{SL}$ and $\mathrm{Y} \times \mathrm{NL}$, respectively, were found to be statistically significant. Statistically significant differences were detected in the interaction genotypeenvironment. The highest average GY was measured in AU_A, followed by AU_B and SI, while the lowest average GY values were measured in AC, followed by FR and GB, respectively (Table 4). SD significantly affected average GY, which increased by $5.4 \%$ from SD200 to SD350 (Table 4). Results of the present study indicated that the SL6.4 treatment increased GY by $8.2 \%$ compared to SL0.

Table 3. Results of the ANOVA for grain yield (GY), hectoliter weight (HW), thousand kernel weight (TKW), protein concentration (PC) and protein yield (PY). The table columns report the Fisher F (F) and the significance levels: ${ }^{*}=0.05,{ }^{* *}=0.01,{ }^{* * *}=0.001, \mathrm{~ns}=$ not significant.

\begin{tabular}{|c|c|c|c|c|c|c|c|c|c|c|c|}
\hline \multirow{2}{*}{$\begin{array}{l}\text { Variability } \\
\text { Sources }\end{array}$} & \multirow[t]{2}{*}{ DF } & \multicolumn{2}{|c|}{$\begin{array}{c}\text { GY } \\
\left(t \text { ha }{ }^{-1}\right)\end{array}$} & \multicolumn{2}{|c|}{$\begin{array}{c}\text { HW } \\
\left(\mathrm{kg} \mathrm{hL}^{-1}\right)\end{array}$} & \multicolumn{2}{|c|}{$\begin{array}{c}\text { TKW } \\
\text { (g) }\end{array}$} & \multicolumn{2}{|c|}{$\begin{array}{l}\text { PC } \\
(\%)\end{array}$} & \multicolumn{2}{|c|}{$\begin{array}{c}P Y \\
\left(\mathrm{~kg} \mathrm{ha}^{-1}\right)\end{array}$} \\
\hline & & $F$ & Sig & F & $\operatorname{sig}$ & F & sig & $F$ & sig & F & sig \\
\hline Year & 1 & 80.00 & $* * *$ & 2.49 & $\mathrm{~ns}$ & 40.60 & $* * *$ & 170.00 & $* * *$ & 32.40 & $* * *$ \\
\hline NL & 2 & 0.40 & ns & 0.53 & ns & 7.91 & $* * *$ & 38.20 & $* * *$ & 4.00 & $* * *$ \\
\hline SL & 1 & 34.20 & $* * *$ & 0.3 & ns & 3.00 & ns & 2.39 & ns & 39.40 & $* * *$ \\
\hline SD & 1 & 15.50 & $* * *$ & 0.17 & $\mathrm{~ns}$ & 0.00 & ns & 15.00 & $* * *$ & 23.10 & $* * *$ \\
\hline Gen & 13 & 32.40 & $* * *$ & 6.54 & $* * *$ & 23.90 & $* * *$ & 10.70 & $* * *$ & 32.80 & $* * *$ \\
\hline $\mathrm{SL} \times \mathrm{SD}$ & 1 & 0.23 & ns & 0.01 & ns & 0.02 & ns & 0.00 & ns & 0.30 & ns \\
\hline $\mathrm{NL} \times \mathrm{SD}$ & 2 & 0.52 & ns & 0.02 & ns & 0.25 & ns & 0.37 & ns & 0.61 & ns \\
\hline $\mathrm{NL} \times \mathrm{SL}$ & 2 & 2.76 & ns & 0.16 & $\mathrm{~ns}$ & 0.80 & ns & 0.17 & ns & 3.02 & $*$ \\
\hline $\mathrm{Y} \times \mathrm{SD}$ & 1 & 9.48 & * & 0.47 & ns & 0.11 & ns & 5.30 & * & 13.40 & $* *$ \\
\hline $\mathrm{Y} \times \mathrm{SL}$ & 1 & 0.24 & ns & 0.23 & ns & 5.79 & * & 1.07 & ns & 0.39 & ns \\
\hline $\mathrm{Y} \times \mathrm{NL}$ & 2 & 1.97 & ns & 0.34 & ns & 0.48 & ns & 2.36 & ns & 2.29 & ns \\
\hline Residuals & 980 & & & & & & & & & & \\
\hline
\end{tabular}

In the present study, Gen was the sole factor affecting HW (Table 4). Furthermore, Gen was the dominant factor for TKW, followed by $\mathrm{Y}$, NL, the second-order interaction $\mathrm{Y} \times \mathrm{SD}$ and SL, respectively (Table 3). Among the 14 varieties, the highest HW was measured in AU_A, followed by AU_B, while the lowest HW was measured in GR_M, followed by VE and IN, respectively (Table 4).

The highest TKW was measured in GR, followed by GR_A and GR_M, while the lowest TKW was measured in AN and VE (Table 4). The TKW values were found to be significantly decreased by $9.6 \%$, with the increase from NL35 to NL135.

According to the ANOVA, the PC was significantly dominated by $\mathrm{Y}$, followed by NL, SD, and Gen, while SL did not have a significant effect (Table 3). On the contrary, SL was the dominant factor for PY, followed by Gen, Y, SD and NL (Table 3). As regards the second-order interaction, only $\mathrm{Y} \times \mathrm{SD}$ affected both $\mathrm{PC}$ and PY, while NL $\times$ SL significantly affected only PY (Table 3). Results indicated that sulfur application (SL6.4) increased PY by $8.7 \%$ with respect to SL0 (Table 4). The highest SD treatment significantly increased the PC and PY values with respect to the control by $1.4 \%$ and $6.6 \%$, respectively. Furthermore, the PC and PY significantly increased by $3.8 \%$ and $4.5 \%$, respectively, from NL35 to NL135. 
Table 4. Grain quality parameter mean values (standard error in brackets) of 14 old common wheat varieties as a function of genotype (Gen), nitrogen (NL) and sulfur fertilization (SL), and seeding density (SD). First-order interactions are provided for SD, NL, SL and Y. Lowercase letters represent the Tukey HSD post hoc test results. The table columns report the significance levels: ${ }^{* *}=0.001, \mathrm{~ns}=$ not significant.

\begin{tabular}{|c|c|c|c|c|c|c|c|c|c|c|}
\hline \multirow{2}{*}{$\begin{array}{l}\text { Variability } \\
\text { Sources }\end{array}$} & \multicolumn{2}{|c|}{$\begin{array}{c}\text { GY } \\
\left(t \text { ha }{ }^{-1}\right)\end{array}$} & \multicolumn{2}{|c|}{$\begin{array}{c}\mathrm{HW} \\
\left(\mathrm{kg} \mathrm{hL}^{-1}\right)\end{array}$} & \multicolumn{2}{|c|}{$\begin{array}{c}\text { TKW } \\
\text { (g) }\end{array}$} & \multicolumn{2}{|c|}{$\begin{array}{l}\text { PC } \\
(\%)\end{array}$} & \multicolumn{2}{|c|}{$\begin{array}{c}\text { PY } \\
\left(\mathrm{kg} \mathrm{ha}^{-1}\right)\end{array}$} \\
\hline & Average & sig & Average & $\operatorname{sig}$ & Average & $\operatorname{sig}$ & Average & sig & Average & sig \\
\hline Gen & & $* * *$ & & $* * *$ & & $* * *$ & & $* * *$ & & $* * *$ \\
\hline $\mathrm{AC}$ & $\begin{array}{c}3.36 \\
(0.15)\end{array}$ & e & $\begin{array}{c}80.1 \\
(0.86)\end{array}$ & $a b$ & $\begin{array}{l}43.57 \\
(0.42)\end{array}$ & e & $\begin{array}{l}15.17 \\
(0.11)\end{array}$ & a & $\begin{array}{l}502.67 \\
(21.67)\end{array}$ & $\mathrm{gh}$ \\
\hline $\mathrm{AN}$ & $\begin{array}{c}4.14 \\
(0.09)\end{array}$ & $\mathrm{bc}$ & $\begin{array}{l}79.33 \\
(0.85)\end{array}$ & $\mathrm{ab}$ & $\begin{array}{l}38.72 \\
(0.34)\end{array}$ & $\mathrm{f}$ & $\begin{array}{c}14.74 \\
(0.1)\end{array}$ & abcd & $\begin{array}{l}612.39 \\
(15.88)\end{array}$ & def \\
\hline AU_A & $\begin{array}{c}5.41 \\
(0.14)\end{array}$ & a & $\begin{array}{l}81.89 \\
(0.77)\end{array}$ & a & $\begin{array}{c}46.7 \\
(0.52)\end{array}$ & abcd & $\begin{array}{l}14.64 \\
(0.12)\end{array}$ & bcd & $\begin{array}{l}788.35 \\
(20.06)\end{array}$ & a \\
\hline AU_B & $\begin{array}{c}5.03 \\
(0.12)\end{array}$ & a & $\begin{array}{l}81.75 \\
(0.91)\end{array}$ & a & $\begin{array}{l}44.52 \\
(0.54)\end{array}$ & de & $\begin{array}{c}14.7 \\
(0.11)\end{array}$ & bcd & $\begin{array}{l}736.59 \\
(16.73)\end{array}$ & $a b$ \\
\hline BI & $4.2(0.14)$ & $\mathrm{bc}$ & $\begin{array}{l}79.07 \\
(0.82)\end{array}$ & $a b$ & $\begin{array}{l}47.06 \\
(0.49)\end{array}$ & abcd & $\begin{array}{l}14.97 \\
(0.07)\end{array}$ & $a b$ & $\begin{array}{l}629.77 \\
(21.6)\end{array}$ & de \\
\hline FR & $\begin{array}{c}3.44 \\
(0.13)\end{array}$ & de & $\begin{array}{l}72.75 \\
(1.12)\end{array}$ & c & $\begin{array}{l}47.73 \\
(0.79)\end{array}$ & $a b$ & $\begin{array}{l}14.18 \\
(0.15)\end{array}$ & e & $\begin{array}{c}483 \\
(17.65)\end{array}$ & $\mathrm{h}$ \\
\hline GB & $\begin{array}{c}3.78 \\
(0.05)\end{array}$ & cde & $\begin{array}{l}79.05 \\
(0.72)\end{array}$ & $a b$ & $\begin{array}{l}47.26 \\
(0.72)\end{array}$ & $a b c$ & $\begin{array}{l}14.92 \\
(0.08)\end{array}$ & $a b c$ & $\begin{array}{l}563.99 \\
(8.35)\end{array}$ & efg \\
\hline GR & $\begin{array}{c}4.39 \\
(0.06)\end{array}$ & $\mathrm{b}$ & $\begin{array}{c}78.13 \\
(0.7)\end{array}$ & $\mathrm{ab}$ & $\begin{array}{l}48.76 \\
(0.53)\end{array}$ & a & $\begin{array}{l}14.53 \\
(0.07)\end{array}$ & bcde & $\begin{array}{c}638.1 \\
(10.51)\end{array}$ & $\mathrm{cd}$ \\
\hline GR_A & $\begin{array}{c}3.87 \\
(0.07)\end{array}$ & $\mathrm{cd}$ & $\begin{array}{c}78.7 \\
(0.59)\end{array}$ & $\mathrm{ab}$ & $\begin{array}{l}48.56 \\
(0.51)\end{array}$ & a & $\begin{array}{l}14.31 \\
(0.11)\end{array}$ & de & $\begin{array}{l}555.01 \\
(11.78)\end{array}$ & $\mathrm{fg}$ \\
\hline GR_M & $\begin{array}{c}3.85 \\
(0.07)\end{array}$ & cde & $\begin{array}{c}77.05 \\
(0.9)\end{array}$ & $\mathrm{b}$ & $\begin{array}{l}48.11 \\
(0.44)\end{array}$ & a & $\begin{array}{l}14.63 \\
(0.09)\end{array}$ & bcd & $\begin{array}{l}564.74 \\
(11.53)\end{array}$ & efg \\
\hline IN & $\begin{array}{c}3.93 \\
(0.08)\end{array}$ & bcd & $\begin{array}{l}77.57 \\
(0.99)\end{array}$ & $b$ & $\begin{array}{l}47.03 \\
(0.81)\end{array}$ & abcd & $\begin{array}{l}14.13 \\
(0.11)\end{array}$ & e & $\begin{array}{l}554.14 \\
(11.78)\end{array}$ & fgh \\
\hline ME & $\begin{array}{c}4.24 \\
(0.15)\end{array}$ & $\mathrm{bc}$ & $\begin{array}{l}79.87 \\
(0.79)\end{array}$ & $\mathrm{ab}$ & $\begin{array}{l}45.15 \\
(0.5)\end{array}$ & bcde & $\begin{array}{l}14.48 \\
(0.13)\end{array}$ & cde & $\begin{array}{l}607.21 \\
(19.84)\end{array}$ & def \\
\hline SI & $\begin{array}{c}4.92 \\
(0.11)\end{array}$ & a & $\begin{array}{l}78.58 \\
(0.94)\end{array}$ & $a b$ & $\begin{array}{l}44.72 \\
(0.55)\end{array}$ & cde & $\begin{array}{l}14.31 \\
(0.09)\end{array}$ & de & $\begin{array}{l}702.15 \\
(15.3)\end{array}$ & $\mathrm{bc}$ \\
\hline VE & $\begin{array}{c}3.84 \\
(0.09)\end{array}$ & cde & $\begin{array}{c}77.33 \\
(1.1)\end{array}$ & $\mathrm{b}$ & $\begin{array}{l}43.69 \\
(0.54)\end{array}$ & e & $\begin{array}{l}14.84 \\
(0.11)\end{array}$ & $a b c$ & $\begin{array}{l}567.13 \\
(12.7)\end{array}$ & defg \\
\hline SD & & $* * *$ & & $\mathrm{~ns}$ & & ns & & $* * *$ & & $* * *$ \\
\hline SD200 & $\begin{array}{c}4.06 \\
(0.05)\end{array}$ & $\mathrm{b}$ & $\begin{array}{l}78.75 \\
(0.35)\end{array}$ & & $\begin{array}{l}45.83 \\
(0.23)\end{array}$ & & $\begin{array}{l}14.51 \\
(0.05)\end{array}$ & $\mathrm{b}$ & $\begin{array}{c}588.12 \\
(6.65)\end{array}$ & $\mathrm{b}$ \\
\hline SD350 & $\begin{array}{c}4.28 \\
(0.05)\end{array}$ & a & $\begin{array}{l}78.56 \\
(0.33)\end{array}$ & & $\begin{array}{l}45.82 \\
(0.25)\end{array}$ & & $\begin{array}{l}14.71 \\
(0.04)\end{array}$ & $\mathrm{a}$ & $\begin{array}{l}626.92 \\
(7.32)\end{array}$ & a \\
\hline $\mathrm{NL}$ & & $\mathrm{ns}$ & & ns & & $* * *$ & & $* * *$ & & $* * *$ \\
\hline NL35 & $\begin{array}{c}4.14 \\
(0.06)\end{array}$ & & $\begin{array}{l}78.97 \\
(0.39)\end{array}$ & & $\begin{array}{l}46.56 \\
(0.3)\end{array}$ & a & $\begin{array}{l}14.34 \\
(0.05)\end{array}$ & c & $\begin{array}{c}591.88 \\
(8.04)\end{array}$ & $\mathrm{b}$ \\
\hline NL80 & $4.2(0.06)$ & & $\begin{array}{l}78.61 \\
(0.45)\end{array}$ & & $\begin{array}{c}45.8 \\
(0.31)\end{array}$ & $a b$ & $\begin{array}{l}14.61 \\
(0.05)\end{array}$ & $\mathrm{b}$ & $\begin{array}{c}611.89 \\
(8.8)\end{array}$ & $a b$ \\
\hline NL135 & $\begin{array}{c}4.17 \\
(0.06)\end{array}$ & & $\begin{array}{l}78.38 \\
(0.41)\end{array}$ & & $\begin{array}{l}45.12 \\
(0.27)\end{array}$ & $\mathrm{b}$ & $\begin{array}{l}14.88 \\
(0.05)\end{array}$ & $\mathrm{a}$ & $\begin{array}{l}618.78 \\
(8.94)\end{array}$ & a \\
\hline SL & & $* * *$ & & $\mathrm{~ns}$ & & ns & & ns & & $* * *$ \\
\hline SL0 & $\begin{array}{c}4.01 \\
(0.05)\end{array}$ & $\mathrm{b}$ & $\begin{array}{l}78.53 \\
(0.31)\end{array}$ & & $\begin{array}{l}45.57 \\
(0.25)\end{array}$ & & $\begin{array}{l}14.57 \\
(0.04)\end{array}$ & & $\begin{array}{c}582.18 \\
(6.7)\end{array}$ & $\mathrm{b}$ \\
\hline SL6.4 & $\begin{array}{c}4.34 \\
(0.05)\end{array}$ & a & $\begin{array}{l}78.78 \\
(0.37)\end{array}$ & & $\begin{array}{l}46.08 \\
(0.24)\end{array}$ & & $\begin{array}{l}14.65 \\
(0.04)\end{array}$ & & $\begin{array}{l}632.85 \\
(7.19)\end{array}$ & a \\
\hline
\end{tabular}


Table 4. Cont.

\begin{tabular}{|c|c|c|c|c|c|c|c|c|c|c|}
\hline \multirow{2}{*}{$\begin{array}{c}\text { Variability } \\
\text { Sources }\end{array}$} & \multicolumn{2}{|c|}{$\begin{array}{c}\mathrm{GY} \\
\left(\mathrm{t} \mathrm{ha}^{-1}\right)\end{array}$} & \multicolumn{2}{|c|}{$\begin{array}{c}\text { HW } \\
\left(\mathrm{kg} \mathrm{hL}^{-1}\right)\end{array}$} & \multicolumn{2}{|c|}{$\begin{array}{c}\text { TKW } \\
\text { (g) }\end{array}$} & \multicolumn{2}{|c|}{$\begin{array}{l}\text { PC } \\
(\%)\end{array}$} & \multicolumn{2}{|c|}{$\begin{array}{c}\text { PY } \\
\left(\mathrm{kg} \mathrm{ha}^{-1}\right)\end{array}$} \\
\hline & Average & sig & Average & sig & Average & $\operatorname{sig}$ & Average & sig & Average & sig \\
\hline $\mathrm{Y}$ & & $* * *$ & & ns & & $* * *$ & & $* * *$ & & $* * *$ \\
\hline 2018 & $\begin{array}{c}3.92 \\
(0.04)\end{array}$ & $\mathrm{b}$ & $\begin{array}{l}79.02 \\
(0.34)\end{array}$ & & $\begin{array}{l}46.77 \\
(0.26)\end{array}$ & a & $\begin{array}{l}14.94 \\
(0.04)\end{array}$ & a & $\begin{array}{c}584.54 \\
(6.48)\end{array}$ & $\mathrm{b}$ \\
\hline 2019 & $\begin{array}{c}4.42 \\
(0.05)\end{array}$ & a & $\begin{array}{l}78.29 \\
(0.34)\end{array}$ & & $\begin{array}{l}44.89 \\
(0.22)\end{array}$ & $\mathrm{b}$ & $\begin{array}{l}14.28 \\
(0.04)\end{array}$ & $\mathrm{b}$ & $\begin{array}{l}630.5 \\
(7.53)\end{array}$ & $\mathrm{a}$ \\
\hline
\end{tabular}

\subsection{Alveograph Parameters and Free Asparagine Content in Whole Flour}

As regards the main factor, NL was the dominant factor for W, followed by SL, SD, Gen and finally $\mathrm{Y}$ in decreasing order, respectively (Table 5). Additionally, W was strongly affected by the second-order interaction $\mathrm{NL} \times \mathrm{SL}$, while no interactions between $\mathrm{Y}$ and the agronomic treatments were detected.

Table 5. Results of the ANOVA for dough strength (W), the ratio between dough tenacity and dough extensibility $(\mathrm{P} / \mathrm{L})$, and ASN concentration in whole flour. The table columns report the Fisher F (F) and the significance levels: ${ }^{*}=0.05,{ }^{* *}=0.01,{ }^{* *}=0.001, \mathrm{~ns}=$ not significant.

\begin{tabular}{|c|c|c|c|c|c|c|c|}
\hline \multirow{2}{*}{$\begin{array}{l}\text { Variability } \\
\text { Sources }\end{array}$} & \multirow[t]{2}{*}{ DF } & \multicolumn{2}{|c|}{$\begin{array}{c}W \\
\left(10^{-4} \mathrm{~J}\right)\end{array}$} & \multicolumn{2}{|c|}{$\mathrm{P} / \mathrm{L}$} & \multicolumn{2}{|c|}{$\begin{array}{c}\text { Asparagine } \\
\left({\left.\text { Micromoles } \mathrm{g}^{-1}\right)}^{-1}\right.\end{array}$} \\
\hline & & F & $\operatorname{sig}$ & F & sig & $F$ & sig \\
\hline Year & 1 & 11.9 & $* *$ & 0.298 & ns & 215.0 & $* * *$ \\
\hline NL & 2 & 446.0 & $* * *$ & 47.9 & $* * *$ & 300.0 & $* * *$ \\
\hline SL & 1 & 77.4 & $* * *$ & 5.66 & * & 3966.0 & $* * *$ \\
\hline SD & 1 & 67.0 & $* * *$ & 35.5 & $* * *$ & 0.0 & ns \\
\hline Gen & 13 & 28.2 & $* * *$ & 62.3 & $* * *$ & 15.9 & $* * *$ \\
\hline $\mathrm{SL} \times \mathrm{SD}$ & 1 & 0.3 & ns & 11.6 & $* * *$ & 0.0 & ns \\
\hline $\mathrm{NL} \times \mathrm{SD}$ & 2 & 3.4 & * & 0.69 & ns & 0.0 & ns \\
\hline $\mathrm{NL} \times \mathrm{SL}$ & 2 & 52.9 & $* * *$ & 38.3 & $* * *$ & 177.0 & $* * *$ \\
\hline $\mathrm{Y} \times \mathrm{SD}$ & 1 & 0.2 & ns & 0.11 & ns & 0.0 & ns \\
\hline $\mathrm{Y} \times \mathrm{SL}$ & 1 & 0.3 & ns & 0.719 & ns & 53.7 & $* * *$ \\
\hline $\mathrm{Y} \times \mathrm{NL}$ & 2 & 0.5 & ns & 0.169 & ns & 6.1 & $* * *$ \\
\hline Residuals & 308 & & & & & & \\
\hline
\end{tabular}

The highest $\mathrm{W}$ was measured in SI, followed by GB and FR, while the lowest values were measured in AN, followed in increasing order by BI, VE, GR_M, and ME, respectively (Table 6). The $\mathrm{W}$ decreased by about $19.3 \%$ as SD increased from SD200 to SD350 (Table 6). In contrast, the $\mathrm{W}$ value increased by $84.4 \%$ and $15.9 \%$ with the NL treatment (from N35 to N135) and the SL treatment, respectively. The S fertilization did not affect the $\mathrm{W}$ at N35, while W increased when S was applied at the NL80 and NL135 treatments, respectively (Figure 3).

Thus, at S0, W increased from $25 \%$ at NL80 to $55.5 \%$ at NL135, while at S6.4, the W increased from $37.4 \%$ at NL80 to $112.7 \%$ at NL135 compared to the lowest N fertilization level. 
Table 6. Averages (standard error in brackets) of dough strength (W), the ratio between dough tenacity and dough extensibility $(\mathrm{P} / \mathrm{L})$, and asparagine concentration in whole flour as a function of genotype (Gen), nitrogen (NL) and sulfur fertilization (SL), seeding density (SD), and first-order interaction. Lowercase letters represent the Tukey HSD post hoc test results. The table columns report the significance levels: ${ }^{* *}=0.01,{ }^{* * *}=0.001, \mathrm{~ns}=$ not significant.

\begin{tabular}{|c|c|c|c|c|c|c|}
\hline \multirow{2}{*}{$\begin{array}{l}\text { Variability } \\
\text { Sources }\end{array}$} & \multicolumn{2}{|c|}{$\begin{array}{c}W \\
\left(10^{-4} \mathrm{~J}\right)\end{array}$} & \multicolumn{2}{|c|}{$\mathrm{P} / \mathrm{L}$} & \multicolumn{2}{|c|}{$\begin{array}{c}\text { Asparagine } \\
\left.\text { (Micromoles g }^{-1}\right)\end{array}$} \\
\hline & Average & sig & Average & sig & Average & sig \\
\hline Gen & & $* * *$ & & $* * *$ & & $* * *$ \\
\hline $\mathrm{AC}$ & $62.74(3.72)$ & $\mathrm{cd}$ & $0.61(0.03)$ & efg & $19.74(3.5)$ & bcd \\
\hline $\mathrm{AN}$ & $52.41(3.42)$ & e & $0.84(0.06)$ & $\mathrm{cd}$ & $19.71(3.45)$ & bcd \\
\hline AU_A & $78.94(5.08)$ & $\mathrm{b}$ & $0.6(0.03)$ & efg & $16.99(3.06)$ & cde \\
\hline AU_B & $78.42(5.77)$ & $\mathrm{b}$ & $0.71(0.02)$ & de & $17.92(2.97)$ & $\mathrm{cd}$ \\
\hline $\mathrm{BI}$ & $55.22(3.41)$ & de & $0.55(0.02)$ & $\mathrm{fg}$ & $16.52(3.03)$ & de \\
\hline FR & $80.7(6.83)$ & $\mathrm{ab}$ & $0.88(0.06)$ & $\mathrm{bc}$ & $17.23(3.22)$ & cde \\
\hline GB & 81.35 (6.25) & $a b$ & $0.71(0.04)$ & de & $23.69(4.39)$ & $a b$ \\
\hline GR & $67.96(2.56)$ & $\mathrm{C}$ & $0.68(0.03)$ & ef & $22.94(3.83)$ & $\mathrm{ab}$ \\
\hline GR_A & $63.5(2.72)$ & $\mathrm{cd}$ & $0.69(0.04)$ & def & $25.12(4.72)$ & $\mathrm{a}$ \\
\hline GR_M & $62.16(3.29)$ & cde & $0.7(0.03)$ & def & $24.52(4.52)$ & $\mathrm{a}$ \\
\hline IN & $63.33(3.68)$ & $\mathrm{cd}$ & $0.52(0.03)$ & $\mathrm{g}$ & $21.01(3.76)$ & $\mathrm{abc}$ \\
\hline ME & $61.97(2.83)$ & cde & $0.71(0.05)$ & de & $17.92(3.11)$ & $\mathrm{cd}$ \\
\hline SI & $89.67(7.16)$ & $\mathrm{a}$ & $1.54(0.07)$ & $\mathrm{a}$ & $13.64(2.43)$ & $\mathrm{e}$ \\
\hline VE & 58.18 (3.69) & cde & $1.03(0.05)$ & $\mathrm{b}$ & $17.1(3.06)$ & cde \\
\hline SD & & $* * *$ & & $* * *$ & & ns \\
\hline SD200 & 73.17 (1.13) & $\mathrm{a}$ & $0.72(0.02)$ & $\mathrm{b}$ & 19.59 (0.77) & \\
\hline SD350 & $63.67(1.01)$ & $\mathrm{b}$ & $0.82(0.01)$ & a & $19.58(0.78)$ & \\
\hline NL & & $* * *$ & & $* * *$ & & $* * *$ \\
\hline NL35 & $49.27(0.48)$ & c & $0.82(0.02)$ & a & $11.66(0.56)$ & c \\
\hline NL80 & $65.16(0.66)$ & $\mathrm{b}$ & $0.84(0.02)$ & a & $22.48(1.01)$ & $\mathrm{b}$ \\
\hline NL135 & $90.83(1.44)$ & $\mathrm{a}$ & $0.65(0.02)$ & $\mathrm{b}$ & $24.6(1.04)$ & a \\
\hline SL & & $* * *$ & & $* *$ & & $* * *$ \\
\hline SLO & $63.37(0.82)$ & $\mathrm{b}$ & $0.79(0.01)$ & a & 34.08 (0.59) & $\mathrm{b}$ \\
\hline SL6.4 & 73.46 (1.27) & $\mathrm{a}$ & $0.75(0.02)$ & $\mathrm{b}$ & $5.08(0.12)$ & $\mathrm{a}$ \\
\hline $\mathrm{Y}$ & & $* * *$ & & $\mathrm{~ns}$ & & $* * *$ \\
\hline 2018 & $70.56(0.79)$ & a & $0.77(0.01)$ & & $16.23(0.67)$ & $\mathrm{b}$ \\
\hline 2019 & $66.28(0.77)$ & $\mathrm{b}$ & $0.77(0.01)$ & & $22.93(0.79)$ & a \\
\hline
\end{tabular}

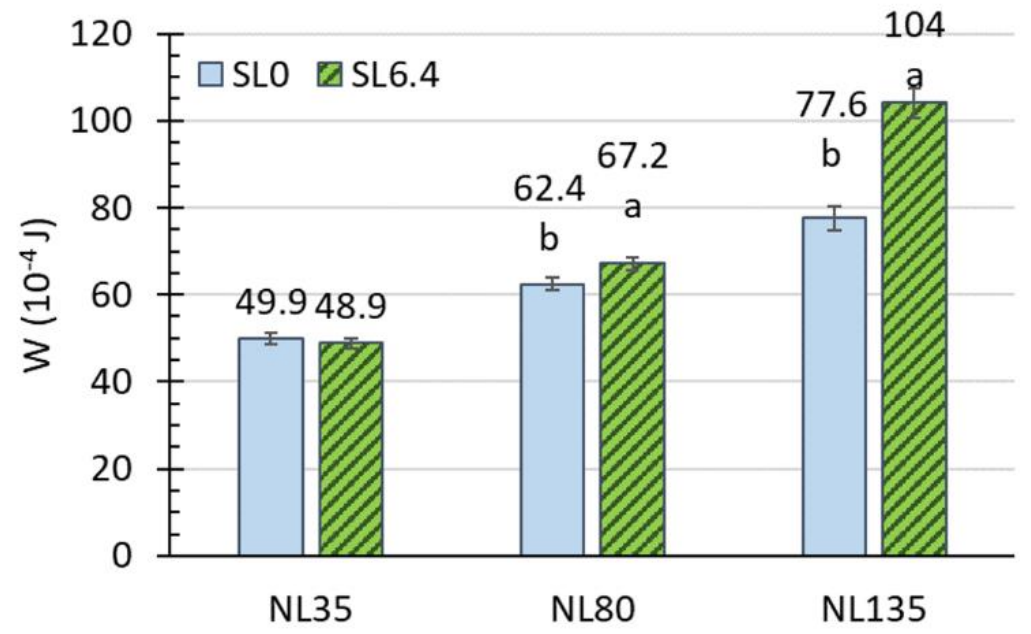

Figure 3. Effect of nitrogen fertilization level (NL) and sulfur fertilization level (SL) on dough strength (W). Lowercase letters represent the Tukey HSD post hoc test results. 
In the present trial, $\mathrm{P} / \mathrm{L}$ was significantly affected by genotype. Of the 14 varieties, 10 had optimal $\mathrm{P} / \mathrm{L}$ ranges, while BI and IN showed lower values, with SI and VE showing higher values, respectively (Table 6). Regardless of the variety, P/L was not affected by $\mathrm{Y}$, highlighting the strong genotype effect on this characteristic. Conversely, agronomical practices affected P/L. The increase in SD significantly increased the P/L (Table 6). As the main effect, SL significantly decreased P/L. However, the SL interactions with SD and NL need to be considered. SL decreased the P/L only at the lower SD, while no significant effect was found at the higher SD (Figure 4). P/L was also decreased at the higher NL, while no significant difference was found between NL35 and NL80. Instead, there was a significant decrease in P/L at NL135 in combination with the SL treatment (Figure 4). Moreover, the $\mathrm{P} / \mathrm{L}$ value was shown to be below the 0.6 threshold with SL and NL135 treatments.
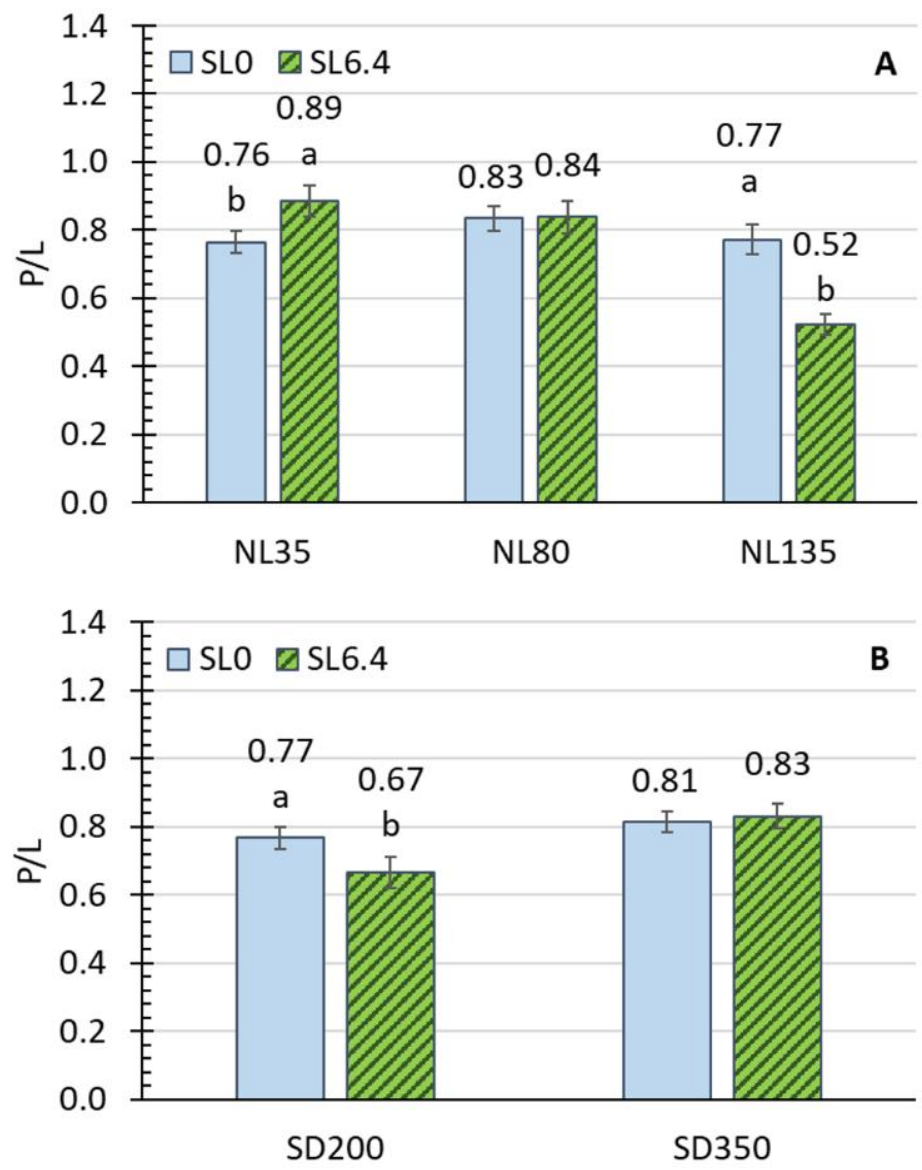

Figure 4. (A) Effect of nitrogen fertilization level (NL) and sulfur fertilization level (SL) on the ratio between dough tenacity and dough extensibility (P/L). (B) Effect of seeding density level (SD) and sulfur fertilization level (SL) on the ratio between dough tenacity and dough extensibility (P/L). Lowercase letters represent the Tukey HSD post hoc test results.

The SL treatment was by far the most important factor influencing the concentration of free ASN in grains, followed by NL, Y, and Gen in decreasing order, respectively (Table 5). $\mathrm{SD}$ was the only agronomic treatment not exerting a significant effect on free ASN concentration. The free ASN concentration in grain was affected by the second-order interaction $\mathrm{NL} \times \mathrm{SL}$, followed by $\mathrm{Y} \times \mathrm{SL}$ and $\mathrm{Y} \times \mathrm{NL}$ (Table 5). Free ASN concentration in grain was significantly higher in 2019 than in 2018. When combining both years, the ASN content significantly increased from $92.8 \%$ at NL80 to $111 \%$ at NL135 compared to N35. Instead, the ASN content was shown to decrease by $85.1 \%$ with the SL treatment. In the present study, S fertilization was more effective in reducing the ASN concentration in 2018 than in 2019 (Figure 5). S treatment decreased the ASN concentration by 7.5 and 4.8 times in 2018 and 2019, respectively. At the same time, during the two growing seasons, $\mathrm{N}$ fertilization 
had a contrasting effect to that of S. In particular, the $\mathrm{N}$ fertilization increased the ASN concentration by 197\% and 72\% in 2018 and 2019, respectively. A more effective reduction in grain ASN concentration was observed at NL80 than at the remaining N fertilization levels (Figure 5). Particularly, the decrease in ASN content measured at SL0 and SL6.4, respectively, was not significantly different between NL35 and NL135 (6.09 and 6.01 times, respectively), while the decrease in ASN was significantly different at NL80 (8.2 times). The highest free asparagine concentration was measured in GR_A, followed by GR_M, GB, and GR, while the lowest values were measured in SI, followed in increasing order by BI, AU_A, VE, and FR, respectfully (Table 6).

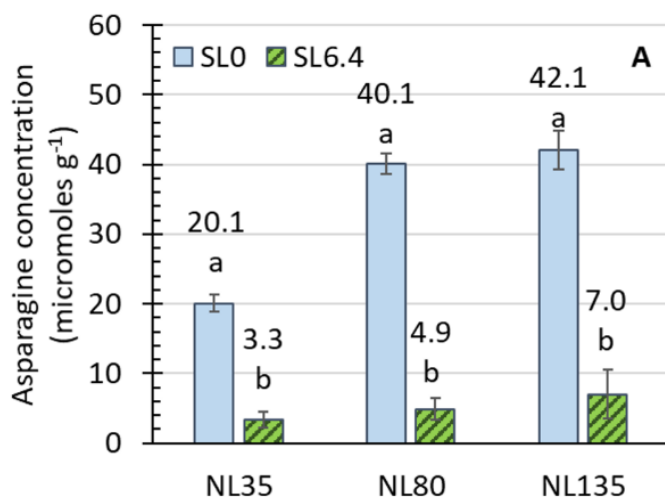

NL35

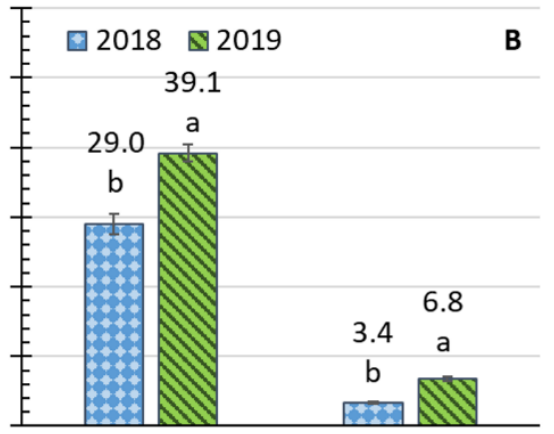

SLO

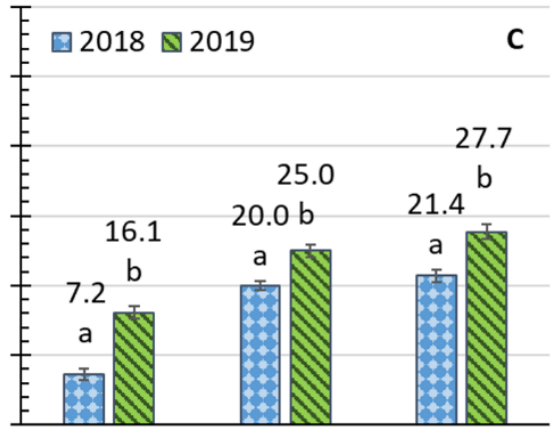

NL35 NL80

NL135

Figure 5. Interactions between years and agronomic treatments on asparagine content in grain; (A) Effect of nitrogen fertilization level (NL) and sulfur fertilization level (SL); (B) effect of year (Y) and sulfur fertilization level (SL); (C) effect of the year (Y) and nitrogen fertilization (NL). Lowercase letters represent the Tukey HSD post hoc test results.

Interestingly, when the average ASN levels determined in the present study were plotted against the date when the varieties were released, there was a significant decline $\left(\mathrm{R}^{2}=0.69, p<0.01\right)$ in ASN content across the release year of the considered varieties (Figure 6).

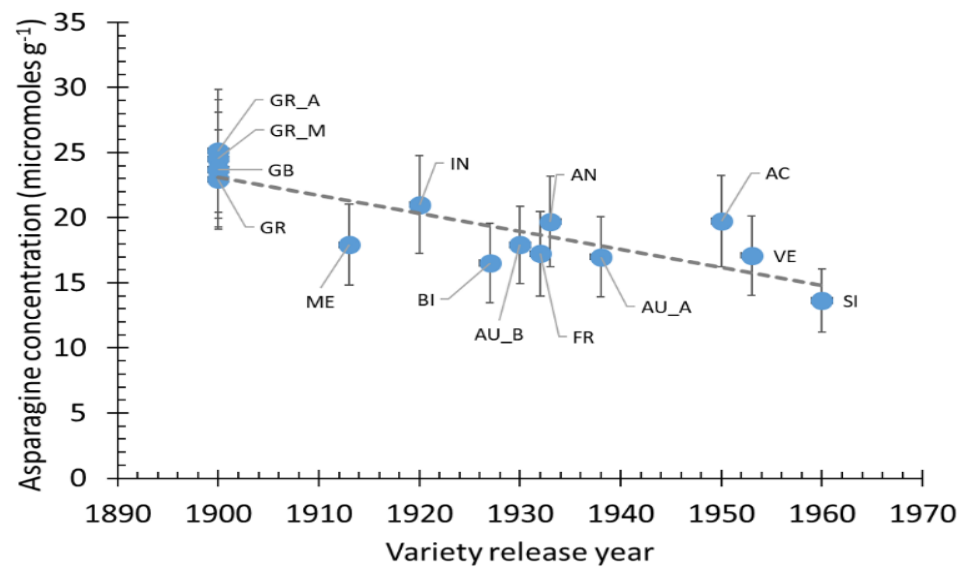

Figure 6. Scatter plots of free asparagine concentration in grain as a function of the variety year of release.

\section{Discussion}

In general, the results suggest that the $\mathrm{N}$ concentration in the soil of the study site was not a limiting factor for the growth and production of these 'old' common wheat genotypes. Gooding et al. (2002) [40] and Zhang et al. (2016) [41] found a significant interaction between $\mathrm{N}$ fertilization and seeding density in determining the kernel yield, whilst no interactions between $\mathrm{SD} \times \mathrm{NL}$, respectively, were found to be statistically significant in this study. Our study corroborated previous results [42], indicating that genotypes having 
high tillering potential may benefit from SD up to 400 seeds $\mathrm{m}^{-2}$. In contrast, Zhang et al. (2016) [41] found that SD increased from 120 to 180 plants $\mathrm{m}^{-2}$, significantly increasing GY, with no further increases observed as SD increased from 180 to 240 plants $\mathrm{m}^{-2}$. The present study suggests that the sulfur treatment can significantly increase GY. However, variable effects in response to sulfur treatment have been reported in previous literature. For example, Wilson et al. (2020) [20] found that foliar application of $20 \mathrm{~kg} \mathrm{Sha}^{-1}$ increased GY by up to 55\% compared to the control. Instead, Guerrini et al. (2020) [7] reported that sulfur treatment did not significantly affect GY of 'old' Italian common wheat landraces. In general, the present results corroborated those of Kilmer and Nearpass (1960) [32], indicating that crops respond to sulfur fertilization in sulfur-deficient soils. Salvagiotti and Miralles (2008) [43] showed that S fertilization increased grain yield in wheat by increasing nitrogen use efficiency (NUE). Further, Salvagiotti et al. (2009) [25] suggested that sulfur fertilization can increase the NUE in sulfur-deficient soils. In the 'old' varieties used in this study, the genotypic factor predominated on the HW and TKW, corroborating previous results for Italian landraces [7]. In contrast, in modern varieties, HW values were shown to increase, with increasing $\mathrm{N}$ up to $150 \mathrm{~kg} \mathrm{ha}^{-1}$ [44]. Our study indicated a strong effect of $\mathrm{N}$ and $\mathrm{S}$ fertilization on PC and PY production. These results were consistent with previous findings in 'modern' common wheat varieties [45-49]. Likewise, Guerrini et al. (2020) [7] reported that $\mathrm{S}$ and $\mathrm{N}$ fertilization substantially affected the PC in 'old' varieties. $\mathrm{Yu}$ et al. (2021) [50] observed a reduced efficiency of sole $\mathrm{N}$ fertilization in increasing both protein and grain yield in sulfur-deficient soils. Further, Yu et al. (2021) [50] suggested that sulfur application can result in protein and grain yield increases by regulating glutamine synthetase 1 and improving nitrogen-use efficiency.

Our results suggest that nitrogen fertilization may be used as a tool to modify the dough deformation energy (i.e., alveograph $\mathrm{W}$ ) in these 'old' varieties and highlight a positive synergy between $\mathrm{N}$ and $\mathrm{S}$. The $\mathrm{W}$ values were consistent with those measured in previous studies $[7,8]$. As 'old' common wheat flours are usually characterized by a low $\mathrm{W}$, any increase in this value can be regarded with interest as it improves the flour's bread-making characteristics $[7,51]$. Therefore, the observed increases in W with the NL and SL treatments, respectively, are of particular interest for 'old' common wheat varieties. The effect of $\mathrm{S}$ and $\mathrm{N}$ fertilization on $\mathrm{W}$ was consistent with those measured previously $[7,52]$. Considering all the varieties, the agronomic treatments were unsuccessful in increasing the $\mathrm{W}$ values above $90 \times 10^{-4} \mathrm{~J}$, which, according to the common classification, distinguishes biscuit flours from flours suitable for bread-making. However, the $90 \times 10^{-4} \mathrm{~J}$ threshold was exceeded by five varieties at NL135 $\left(132.3,118.4,117.8,110.7\right.$, and $108.6 \times 10^{-4} \mathrm{~J}$ in SI, GB, FR, AU_B, and AU_A, respectively), thus attaining the status of weak flours, attributable to this level of nitrogen fertilization. A P/L range of $0.6-0.8$ is usually considered the optimal ratio between dough tenacity and extensibility (i.e., $\mathrm{P} / \mathrm{L}$ ) in bread-making flours [53]. P/L ratios exceeding 0.8 are known to be lacking in old varieties for bread-making as unrefined flours [51]. SI and VE have been extensively studied in the literature and are popular among bakers using flour from 'old' varieties, already known for high tenacity and low extensibility doughs [51]. In the literature, there has been speculation on the advantages of a blending strategy between the "poor" $\mathrm{P} / \mathrm{L}$ wheats, such as BI and IN, and the most commonly used higher P/L wheats (SI and VE) in order to improve the bread-making performances, thereby promoting the valorization of local germplasm characteristics $[7,51]$. The dough parameters highlight the importance of agronomical practices in modulating the technological performance of dough in old, weak varieties. Old varieties are widely reported as having weaker dough, with unbalanced tenacity-extensibility ratios, rendering baking difficult. Hence, the effect of agronomical practices on dough strength necessitates investigation, with careful selection of SD, NL, and SL to optimize rheological parameters for the baking industry. The ASN concentration determined in 2019 was higher than in 2018. This was attributable to the stress incurred by the higher temperatures combined with lower precipitation over the entire growing season and, in particular, during the grainfilling stage. Similar interactions between ASN content and environmental stress conditions 
were also reported previously [20]. Results indicated that the $\mathrm{N}$ fertilization increased the ASN content, while sulfur fertilization was able to reduce the ASN content by up to $85.1 \%$. This result was consistent with that observed by Wilson et al. (2020) [20], showing an increase in ASN content in response to increasing N. Moreover, present results were similarly consistent with various studies reporting higher ASN contents in wheat grains cultivated in sulfur-deficient soils $[4,54]$. In contrast, in soil with satisfactory $S$ availability, $S$ fertilization does not impact on the ASN content in grain [29,55]. Previously, it was noted that in three 'old' common wheat varieties (namely, AN, SI and VE), the albumin, globulin, and gliadin fractions were decreased significantly, whilst the glutenin fraction was significantly increased in response to $S$ fertilization [7]. Thus, it could be possible that these 'old' common wheat varieties were highly responsive to $S$ deficiency and that changes in the protein composition resulted in a significant increase in ASN content. The ASN content was consistent with that measured previously for wheat [20,30]. Poudel et al. (2021) [30] suggested that despite the absence of a legal limit for ASN concentrations in grain, this should be as low as possible. This is the first time that a negative correlation between the ASN content and the release year has been shown for old Italian common wheat varieties. Furthermore, significant correlations between free ASN and grain protein content were reported previously and shown to be higher in the old varieties [56]. Corol et al. (2016) [19] reported a weak correlation between ASN concentration and the release year. However, those authors also found that free ASN content was positively correlated to plant height [19], which, interestingly, is generally higher in the old varieties. In contrast, more recent work, analyzing the free ASN content in grain of 19 cultivars released between 1870 and 2013 across two growing seasons in the USA, showed that the free ASN concentration in grain was significantly increased in the second growing season across the release years, whilst no trend across release year was detected during the first growing season [30]. Given the scarcity of information, the requisite for further investigating this aspect in future research programs is evidenced. Consequently, further studies involving a larger number of genotypes over a longer breeding period should be conducted to provide additional insights into the effect of previous breeding programs on the compositional properties of 'old' common wheat varieties. Nonetheless, the preliminary results suggest that breeding programs may have inadvertently selected against free ASN content. Overall, selection by breeding programs has improved nutrient-use efficiency, increased resistance to lodging by reducing the plant height, as well as resistance to stress conditions such as water stagnation, drought, and plant diseases, which are notorious for affecting the ability of wheat to convert assimilated nitrogen $(\mathrm{N})$ into free amino acids and then proteins [20].

\section{Conclusions}

This paper was aimed at evaluating whether the grain yield and protein, rheological characteristics, as well as the ASN content in kernels of 'old' common wheat varieties grown on S-deficient soils could be improved with agronomical treatments, more specifically $S$ fertilization, $\mathrm{N}$ fertilization, and SD. The experiment was conducted on 14 'old' common wheat varieties released between 1900 to 1960 in Italy. A higher seeding density was shown to increase the grain yield and protein concentration. $S$ fertilization was found to increase the grain yield without decreasing grain protein concentration, while $\mathrm{N}$ fertilization was found to effectively increase the grain protein concentration and the protein yield by hectare. Regarding the dough rheological parameters, SD was shown to negatively affect the dough strength in all the varieties. Instead, dough strength was significantly increased in relation to increasing $\mathrm{S}$ and $\mathrm{N}$ fertilization. Free ASN concentration in 'old' common wheat varieties was found to be comparable to other studies investigating 'old' and 'modern' genotypes with low nitrogen-use efficiency under S-deficient conditions. Interestingly, free ASN concentration was negatively correlated with the year of release in the considered varieties. This may suggest that past breeding programs may have contributed to reducing the ASN content; however, more studies on old varieties need to be conducted to further investigate this aspect. $\mathrm{N}$ fertilization was found to significantly increase the ASN content, whereas 
S application decreased the ASN content by $85.1 \%$. In the present study, S fertilization successfully improved the grain yield and the technical parameters of the 'old' common wheat varieties while reducing the ASN concentration, thereby promoting food safety. Hence, these present results can be considered of particular interest for 'old' common wheat varieties characterized by poor technical performance when these varieties are grown on S-deficient soils. However, additional trials, including additional years within differing pedo-climatic conditions, are required in order to further evaluate the interaction between cultivars and the agronomical treatments.

\begin{abstract}
Author Contributions: Conceptualization, M.N. and L.G.; methodology, M.N. and L.G.; validation, M.N.; formal analysis, M.N. and L.G.; investigation, C.F., M.M., M.N. and L.G.; resources, M.M.; data curation, C.F., M.M., M.N., A.P. and Q.S.; writing-original draft preparation, M.N., A.P., Q.S., C.F. and L.G.; writing-review and editing, M.N. and L.G.; visualization, M.N.; supervision, M.N. and S.O.; project administration, M.N. and M.M.; funding acquisition, S.O. All authors have read and agreed to the published version of the manuscript.
\end{abstract}

Funding: This research was partly funded by MIPAAF Italy Systemic-1063 “An integrated approach to the challenge of sustainable food systems: adaptive and mitigatory strategies to address climate change and malnutrition".

Acknowledgments: The authors want to thank "Tenuta di Cesa" and "Ente Terre Regionali Toscane" for making the fields, the equipment, and, above all, the technical experience of their staff available. A special thanks to Anne Whittaker (Department of Agri-Food Sciences and Technologies, University of Bologna) for English editing and to Roberto Vivoli (Department of Agriculture, Food, Environment and Forestry, University of Florence) for his technical support during the whole experiment.

Conflicts of Interest: The authors declare no conflict of interest. The funders had no role in the design of the study; in the collection, analyses, or interpretation of data; in the writing of the manuscript, or in the decision to publish the results.

\title{
References
}

1. Shewry, P.R.; Hey, S.J. The contribution of wheat to human diet and health. Food Energy Secur. 2015, 4, 178-202. [CrossRef] [PubMed]

2. Poole, N.; Donovan, J.; Erenstein, O. Viewpoint: Agri-nutrition research: Revisiting the contribution of maize and wheat to human nutrition and health. Food Policy 2021, 100, 101976. [CrossRef] [PubMed]

3. FAO. FAO Cereal Supply and Demand Brief I World Food Situation I Food and Agriculture Organization of the United Nations. Available online: https:/ / www.fao.org/worldfoodsituation/csdb/en/ (accessed on 15 December 2021).

4. Shewry, P.R. Wheat. J. Exp. Bot. 2009, 60, 1537-1553. [CrossRef] [PubMed]

5. Arshad, M.A. A Review on Wheat Management, Strategies, Current Problems and Future Perspectives. Haya Saudi J. Life Sci. 2021, 6, 14-18. [CrossRef]

6. Sanchez-Garcia, M.; Álvaro, F.; Peremarti, A.; Martín-Sánchez, J.A.; Royo, C. Changes in bread-making quality attributes of bread wheat varieties cultivated in Spain during the 20th century. Eur. J. Agron. 2015, 63, 79-88. [CrossRef]

7. Guerrini, L.; Napoli, M.; Mancini, M.; Masella, P.; Cappelli, A.; Parenti, A.; Orlandini, S. Wheat grain composition, dough rheology and bread quality as affected by nitrogen and sulfur fertilization and seeding density. Agronomy 2020, 10, 233. [CrossRef]

8. Migliorini, P.; Spagnolo, S.; Torri, L.; Arnoulet, M.; Lazzerini, G.; Ceccarelli, S. Agronomic and quality characteristics of old, modern and mixture wheat varieties and landraces for organic bread chain in diverse environments of northern Italy. Eur. J. Agron. 2016, 79, 131-141. [CrossRef]

9. Sylvester-Bradley, R.; Kindred, D.R. Analysing nitrogen responses of cereals to prioritize routes to the improvement of nitrogen use efficiency. J. Exp. Bot. 2009, 60, 1939-1951. [CrossRef]

10. Cappelli, A.; Cini, E.; Guerrini, L.; Masella, P.; Angeloni, G.; Parenti, A. Predictive models of the rheological properties and optimal water content in doughs: An application to ancient grain flours with different degrees of refining. J. Cereal Sci. 2018, 83, 229-235. [CrossRef]

11. Godfray, H.C.J.; Garnett, T. Food security and sustainable intensification. Philos. Trans. R. Soc. B Biol. Sci. 2014, $369,20120273$. [CrossRef]

12. Suchowilska, E.; Wiwart, M.; Borejszo, Z.; Packa, D.; Kandler, W.; Krska, R. Discriminant analysis of selected yield components and fatty acid composition of chosen Triticum monococcum, Triticum dicoccum and Triticum spelta accessions. J. Cereal Sci. 2009, 49, 310-315. [CrossRef]

13. Dinu, M.; Whittaker, A.; Pagliai, G.; Benedettelli, S.; Sofi, F. Ancient wheat species and human health: Biochemical and clinical implications. J. Nutr. Biochem. 2018, 52, 1-9. [CrossRef] [PubMed] 
14. Dinelli, G.; Segura-Carretero, A.; Di Silvestro, R.; Marotti, I.; Arráez-Román, D.; Benedettelli, S.; Ghiselli, L.; Fernadez-Gutierrez, A. Profiles of phenolic compounds in modern and old common wheat varieties determined by liquid chromatography coupled with time-of-flight mass spectrometry. J. Chromatogr. A 2011, 1218, 7670-7681. [CrossRef] [PubMed]

15. European Commission Dietary Recommendations for Whole Grain Intake I Knowledge for Policy. Available online: https:// knowledge4policy.ec.europa.eu/health-promotion-knowledge-gateway/whole-grain-dietary-recommendations-4_en (accessed on 15 December 2021).

16. Žilić, S.; Aktağ, I.G.; Dodig, D.; Filipović, M.; Gökmen, V. Acrylamide formation in biscuits made of different wholegrain flours depending on their free asparagine content and baking conditions. Food Res. Int. 2020, 132, 109109. [CrossRef] [PubMed]

17. Seal, C.J.; de Mul, A.; Eisenbrand, G.; Haverkort, A.J.; Franke, K.; Lalljie, S.P.D.; Mykkänen, H.; Reimerdes, E.; Scholz, G.; Somoza, V.; et al. Risk-Benefit Considerations of Mitigation Measures on Acrylamide Content of Foods-A Case Study on Potatoes, Cereals and Coffee. Br. J. Nutr. 2008, 99, S1-S46. [CrossRef] [PubMed]

18. IARC Acrylamide. IARC Monographs on the Evaluation of Carcinogenic Risks to Humans; Humans IARCPress: Lyon, France, 1994; Volume 60, pp. 389-433.

19. Corol, D.I.; Ravel, C.; Rakszegi, M.; Charmet, G.; Bedo, Z.; Beale, M.H.; Shewry, P.R.; Ward, J.L. 1H-NMR screening for the high-throughput determination of genotype and environmental effects on the content of asparagine in wheat grain. Plant Biotechnol. J. 2016, 14, 128-139. [CrossRef]

20. Wilson, T.L.; Guttieri, M.J.; Nelson, N.O.; Fritz, A.; Tilley, M. Nitrogen and sulfur effects on hard winter wheat quality and asparagine concentration. J. Cereal Sci. 2020, 93, 102969. [CrossRef]

21. Lea, P.J.; Sodek, L.; Parry, M.A.J.; Shewry, P.R.; Halford, N.G. Asparagine in plants. Ann. Appl. Biol. 2007, 150, 1-26. [CrossRef]

22. Sinclair, T.R.; Horie, T. Leaf Nitrogen, Photosynthesis, and Crop Radiation Use Efficiency: A Review. Crop Sci. 1989, 29, 90-98. [CrossRef]

23. Gyori, Z. Sulphur Content of Winter Wheat Grain in Long Term Field Experiments. Commun. Soil Sci. Plant Anal. 2005, 36, 373-382. [CrossRef]

24. Martinek, P.; Klem, K.; Váňová, M.; Bartáčková, V.; Večerková, L.; Bucher, P.; Hajšlová, J. Effects of nitrogen nutrition, fungicide treatment and wheat genotype on free asparagine and reducing sugars content as precursors of acrylamide formation in bread. Plant Soil Environ. 2009, 55, 187-195. [CrossRef]

25. Salvagiotti, F.; Castellarín, J.M.; Miralles, D.J.; Pedrol, H.M. Sulfur fertilization improves nitrogen use efficiency in wheat by increasing nitrogen uptake. Field Crops Res. 2009, 113, 170-177. [CrossRef]

26. Curtis, T.Y.; Powers, S.J.; Wang, R.; Halford, N.G. Effects of variety, year of cultivation and sulphur supply on the accumulation of free asparagine in the grain of commercial wheat varieties. Food Chem. 2018, 239, 304-313. [CrossRef] [PubMed]

27. De Santis, M.A.; Giuliani, M.M.; Giuzio, L.; De Vita, P.; Flagella, Z. Assessment of grain protein composition in old and modern Italian durum wheat genotypes. Ital. J. Agron. 2018, 13, 40-43. [CrossRef]

28. Navrotskyi, S.; Baenziger, P.S.; Regassa, T.; Guttieri, M.J.; Rose, D.J. Variation in asparagine concentration in Nebraska wheat. Cereal Chem. 2018, 95, 264-273. [CrossRef]

29. Rapp, M.; Schwadorf, K.; Leiser, W.L.; Würschum, T.; Longin, C.F.H. Assessing the variation and genetic architecture of asparagine content in wheat: What can plant breeding contribute to a reduction in the acrylamide precursor? Theor. Appl. Genet. 2018, 131, 2427-2437. [CrossRef]

30. Poudel, R.; Bhinderwala, F.; Morton, M.; Powers, R.; Rose, D.J. Metabolic profiling of historical and modern wheat cultivars using proton nuclear magnetic resonance spectroscopy. Sci. Rep. 2021, 11, 3080. [CrossRef]

31. Olsen, S.R.; Cole, C.V.; Watanabe, F.S.; Dean, L.A. Estimation of Available Phosphorus in Soils by Extraction with Sodium Bicarbonate; USDA Circular: Washington, DC, USA, 1954.

32. Kilmer, V.J.; Nearpass, D.C. The Determination of Available Sulfur in Soils. Soil Sci. Soc. Am. J. 1960, 24, 337-340. [CrossRef]

33. Tuscany Region Seeds and Germplasm Bank of Tuscany Region. Available online: http://germoplasma.regione.toscana.it/ (accessed on 16 December 2021).

34. ISO 7971-1; Cereals—Determination of Bulk Density, Called Mass per Hectolitre-Part 1: Reference Method. ISO: Geneva, Switzerland, 2009.

35. ISO 520; Cereals and Pulses-Determination of the Mass of 1000 Grains. ISO: Geneva, Switzerland, 2010.

36. Žilić, S.; Barać, M.; Pešić, M.; Dodig, D.; Ignjatović-Micić, D. Characterization of proteins from grain of different bread and durum wheat genotypes. Int. J. Mol. Sci. 2011, 12, 5878-5894. [CrossRef]

37. ICC Standard 167; Determination of Crude Protein in Grain and Grain Products for Food and Feed by the Dumas Combustion Principle. International Association for Cereal Science and Technology: ICC: Wien, Austria, 2000.

38. Lecart, B.; Jacquet, N.; Anseeuw, L.; Renier, M.; Njeumen, P.; Bodson, B.; Vanderschuren, H.; Richel, A. Nonconventional enzymatic method to determine free asparagine level in whole-grain wheat. Food Chem. 2018, 251, 64-68. [CrossRef]

39. ISO 27971; Cereals and Cereal Products-Common Wheat (Triticum aestivum L.)—Determination of Alveograph Properties of Dough at Constant Hydration from Commercial or Test Flours and Test Milling Methodology. ISO: Geneva, Switzerland, 2015.

40. Gooding, M.J.; Pinyosinwat, A.; Ellis, R.H. Responses of wheat grain yield and quality to seed rate. J. Agric. Sci. 2002, 138, 317-331. [CrossRef]

41. Zhang, Y.; Dai, X.; Jia, D.; Li, H.; Wang, Y.; Li, C.; Xu, H.; He, M. Effects of plant density on grain yield, protein size distribution, and breadmaking quality of winter wheat grown under two nitrogen fertilisation rates. Eur. J. Agron. 2016, 73, 1-10. [CrossRef] 
42. Valério, I.P.; de Carvalho, F.I.F.; Benin, G.; da Silveira, G.; da Silva, J.A.G.; Nornberg, R.; Hagemann, T.; de Luche, H.S.; de Oliveira, A.C. Seeding density in wheat: The more, the merrier? Sci. Agric. 2013, 70, 176-184. [CrossRef]

43. Salvagiotti, F.; Miralles, D.J. Radiation interception, biomass production and grain yield as affected by the interaction of nitrogen and sulfur fertilization in wheat. Eur. J. Agron. 2008, 28, 282-290. [CrossRef]

44. Litke, L.; Gaile, Z.; Ruza, A. Effect of nitrogen fertilization on winter wheat yield and yield quality. Agron. Res. 2018, 16, 500-509.

45. Khalil, J.K.; Sawaya, W.N.; Hamdallah, G.; Devi Prasad, J. Effect of nitrogen fertilization on the nutritional quality of wheat variety, Yecora Rojo. Plant Foods Hum. Nutr. 1987, 36, 279-293. [CrossRef]

46. Yu, Z.; Juhasz, A.; Islam, S.; Diepeveen, D.; Zhang, J.; Wang, P.; Ma, W. Impact of mid-season sulphur deficiency on wheat nitrogen metabolism and biosynthesis of grain protein. Sci. Rep. 2018, 8, 2499. [CrossRef]

47. Saint Pierre, C.; Peterson, C.J.; Ross, A.S.; Ohm, J.B.; Verhoeven, M.C.; Larson, M.; Hoefer, B. White Wheat Grain Quality Changes with Genotype, Nitrogen Fertilization, and Water Stress. Agron. J. 2008, 100, 414-420. [CrossRef]

48. Luo, C.; Branlard, G.; Griffin, W.B.; McNeil, D.L. The Effect of Nitrogen and Sulphur Fertilisation and their Interaction with Genotype on Wheat Glutenins and Quality Parameters. J. Cereal Sci. 2000, 31, 185-194. [CrossRef]

49. Johansson, E.; Prieto-Linde, M.L.; Svensson, G. Influence of nitrogen application rate and timing on grain protein composition and gluten strength in Swedish wheat cultivars. J. Plant Nutr. Soil Sci. 2004, 167, 345-350. [CrossRef]

50. Yu, Z.; She, M.; Zheng, T.; Diepeveen, D.; Islam, S.; Zhao, Y.; Zhang, Y.; Tang, G.; Zhang, Y.; Zhang, J.; et al. Impact and mechanism of sulphur-deficiency on modern wheat farming nitrogen-related sustainability and gliadin content. Commun. Biol. 2021, 4, 945. [CrossRef] [PubMed]

51. Parenti, O.; Guerrini, L.; Cavallini, B.; Baldi, F.; Zanoni, B. Breadmaking with an old wholewheat flour: Optimization of ingredients to improve bread quality. LWT 2020, 121, 108980. [CrossRef]

52. Tea, I.; Genter, T.; Violleau, F.; Kleiber, D. Changes in the glutathione thiol-disulfide status in wheat grain by foliar sulfur fertilization: Consequences for the rheological properties of dough. J. Cereal Sci. 2005, 41, 305-315. [CrossRef]

53. Cappelli, A.; Guerrini, L.; Parenti, A.; Palladino, G.; Cini, E. Effects of wheat tempering and stone rotational speed on particle size, dough rheology and bread characteristics for a stone-milled weak flour. J. Cereal Sci. 2020, 91, 102879. [CrossRef]

54. Muttucumaru, N.; Halford, N.G.; Elmore, J.S.; Dodson, A.T.; Parry, M.; Shewry, P.R.; Mottram, D.S. Formation of High Levels of Acrylamide during the Processing of Flour Derived from Sulfate-Deprived Wheat. J. Agric. Food Chem. 2006, 54, 8951-8955. [CrossRef]

55. Claus, A.; Schreiter, P.; Weber, A.; Graeff, S.; Herrmann, W.; Claupein, W.; Schieber, A.; Carle, R. Influence of Agronomic Factors and Extraction Rate on the Acrylamide Contents in Yeast-Leavened Breads. J. Agric. Food Chem. 2006, 54, 8968-8976. [CrossRef] [PubMed]

56. Ohm, J.B.; Mergoum, M.; Simsek, S. Variation of Free Asparagine Concentration and Association with Quality Parameters for Hard Red Spring Wheat Grown in North Dakota. Cereal Chem. 2017, 94, 712-716. [CrossRef] 